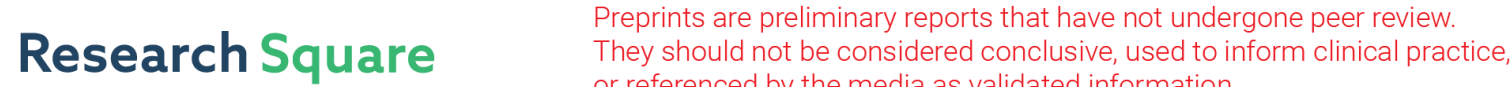 or referenced by the media as validated information. \\ Relationship among foveal avascular zone area, vessel density and foveal ganglion cell layer thickness changes in diabetic retinopathy
}

\section{Xiang-ning Wang}

Shanghai Jiaotong University Affiliated Sixth People's Hospital

\section{Shu-ting Li}

Shanghai Jiaotong University Affiliated Sixth People's Hospital

\section{Xin-hua Du}

Shanghai Jiaotong University Affiliated Sixth People's Hospital

Qiang Wu ( $\Delta$ qiang.Wu@shsmu.edu.cn)

Shanghai Jiao Tong University Affiliated sixth people Hospital https://orcid.org/0000-0002-9169-3791

Research article

Keywords: SD-OCT, Foveal avascular zone, vessel density, ganglion cell layer, Diabetic retinopathy

Posted Date: May 26th, 2019

DOI: https://doi.org/10.21203/rs.2.9856/v1

License: (c) This work is licensed under a Creative Commons Attribution 4.0 International License. Read Full License 


\section{Abstract}

Background To (i) evaluate the variations in the foveal avascular zone area (FAZ), vessel density (VD) and foveal ganglion cell layer (GCC) thickness changes in diabetic retinopathy (DR), as demonstrated by OCT angiography (OCTA) and (ii) correlate these variations. Methods This study was a retrospective observational case series of patients with DR. We studied a total of 64 eyes from patients with diabetic mellitus (DM), including 34 eyes with DR (DR group) and 30 eyes with DM without DR (NDR group). Additionally, 20 age-matched normal people were included as the control group. OCTA was performed using an NIDEK RS-3000 Advance device. Retinal capillary VD, FAZ area and GCC thickness were measured in the foveal regions $(3 * 3 \mathrm{~mm}$ ). Results The FAZ areas in the superficial (SRL) and deep capillary plexus layer $(\mathrm{DL})$ were significantly enlarged in both the NDR and DR groups compared with the control group $(p<0.05)$. In both the NDR and DR groups, the mean VD\% in the SRL/DL, the SupGCC and InfGCC thickness were significantly decreased compared with the control group $(P<0.05)$. No significant difference was observed between the NDR and DR groups for these measurements. Multivariable linear regression showed that the AvgGCC thickness was correlated with FAZ area $(b=-11.372, p=0.001)$ and VD\% $(b=0.752, p=0.007)$ in the DL. Conclusions The FAZ area, mean VD\% and GCC thickness in DR patients were differed compared with healthy controls. The AvgGCC thickness was correlated with FAZ area and VD\% in the DL.

\section{Background}

Diabetic retinopathy (DR) is the dominant cause of vision loss in the working-age population globally[1] . Fluorescein angiography (FA) is the gold standard for evaluating the retinal vasculature. This technique is typically used to evaluate both morphologic and functional variations in the blood vessels in DR[2] . A recent fast, non-invasive technology using optical coherence tomography angiography (OCTA) had enabled us to obtain high-resolution images of retinal and choroidal microvasculature blood flow and structure within seconds[3] .

The foveal avascular zone (FAZ) is the entry point of visual acuity that is histologically a region containing exclusively photoreceptors (cones) with elongated outer segments underlying a capillary-free zone[4]. Studies of diabetic patients, using OCTA, suggest an enlargement and disintegrity of the vascular arcades of the FAZ and visible areas of reduced capillary density $[5,6]$. The retinal ganglion cell complex (GCC) consists of the following three-layer structure: the retinal plexiform layer, the Ganglion cell layer (composed of ganglion cell body), and the retinal nerve fiber layer (by ganglion cell axon constitute) [7] . Studies have indicated that vascular tissue accounts for only $5 \%$ of the entire retinal tissue and that nerve tissue accounts for $95 \%$ of the entire retina[8]. The changes in nerve tissue in the course of DR have been given more attention. Large studies have shown that the effect of DR on the fundus is not only vascular disease but also the degeneration of the nerve, which involves various types of cells on the retina[9] . It was studied that in the early DR, the retinal ganglion cells can change, manifesting as Ganglion cell atrophy and apoptosis, which occurring before neurodegeneration in the Early Stages of $\mathrm{DR}[10]$. 
In this study, we want to evaluate the FAZ area, macular vessel density (VD) and GCC thickness in the early stages of DR using OCTA measurement and to determine whether there is a relationship between them.

\section{Methods}

This study adhered to the tenets of the Declaration of Helsinki and had the approval of the Ethics Committee of Sixth People's Hospital Affiliated to Shanghai Jiao Tong University, Shanghai, China. It was registered in the Chinese clinical trial registry (http://www.chictr.org.cn/,Registration number: ChiCTROOC-16010160). All of the procedures followed the tenets of the Declaration of Helsinki, and informed consent was obtained from all of the subjects before participation in the study.

\section{Patients}

All patients underwent complete ophthalmic examinations, including a detailed fundus examination (TRC-NW300; Topcon, Tokyo, Japan) measurements of manifest refraction, axial length, best-corrected VA (BCVA), slit-lamp biomicroscopy, a detailed fundus examination, SD-OCT (Spectralis HRA+OCT; Heidelberg Engineering, Heidelberg, Germany) and OCTA (NIDEK RS-3000 Advance device; Nidek, Gamagori, Japan). Except the control group, diabetic patients underwent FFA to judge their DR stage, according to the international clinical classification systems for diabetic retinopathy[11] . Only one eye in each participant was selected for this study, generally the eye with the better BCVA or less refractive error was preferred.

The exclusion criteria were as follows: (1) systemic diseases other than type $2 \mathrm{DM}$; (2) pregnant or nursing; (3) a refractive error exceed -6.00 diopters or +3 diopter-equivalent spheres; (4) cannot gave informed consent or complete these examinations; (5) a disease influence the retinal function, including glaucoma, eye injury, vitreous hemorrhage, detachment of retina, age-related macular degeneration, and so on; (6) presence of diabetic macular edema (DME). Age-matched control subjects with no DM and no ocular disease were recruited.

\section{Patient Characteristics}

This study evaluated a total of 64 eyes from 64 patients. There were 30 right eyes and 34 left eyes. The patients were divided into three groups. The DR group (34 eyes) included patients diagnosed with diabetic retinopathy, and the NDR group (30 eyes) included patients diagnosed with diabetic mellitus without diabetic retinopathy. There were 22 patients with mild nonproliferative diabetic retinopathy (NPDR) and 12 with moderate NPDR in the DR group.

There were also 20 age-matched normal people in the control group, and they had never been diagnosed with diabetic mellitus. The number of eyes and patients, and the characteristics of the patients (age, gender, laterality, BMI, BCVA, $\mathrm{HbA} 1 \mathrm{c}$ level, axial length and refractive error) in each group are summarized 
in Table 1. The mean age, refractive error and axial length were $62.4 \pm 10.6,-0.5 \pm 1.1$ diopters spherical equivalent and $23.7 \pm 1.1 \mathrm{~mm}$, respectively, with no significant differences among the three groups $(P=0.537, P=0.463$ and $P=0.316)$. As expected, $B C V A$ was significantly reduced in eyes with $D R(P<$ $0.001)$ compared with the control eyes. In addition, DMEs had significantly higher $\mathrm{HbA} 1 \mathrm{c}$ levels than NonDMEs did $(p<0.001)$.

Table 1. Characteristics of Patients

\section{Optical Coherence Tomography Angiograph}

OCTA was performed with an NIDEK RS-3000 Advance device. The instrument has an scan beam centered at $880 \mathrm{~nm}$, with a bandwidth of $45 \mathrm{~nm}$. This device's A-scan rate is 53,000 scans per second. Each OCT angiography volume consisted of $256 \times 256$ A-scans, with two consecutive B-scans (M-B frames) captured at each fixed position. Two $3 \mathrm{D}$ scans, with a size of $3 * 3 \mathrm{~mm}$, including two horizontal priority ( $x$-fast) scans and one vertical priority ( $y$-fast) scans, were acquired in one session (Fig.1A-B). The $3 \mathrm{D}$ orthogonal registration algorithm was applied to corrected the motion artifacts and flowing blood from static tissue were distinguished using a SSADA algorithm. The internal software (V.2015.1.0.90) automatically divides these scans into an en face macular angiogram of four default segmentations. Only good quality OCT angiograms, as defined by a signal strength index of $\geq 8$, were used for research.

The following parameters in this region were evaluated: superficial VD (\%) and superficial FAZ area (mm $\left.{ }^{2}\right)$, deep VD (\%,) and deep FAZ area ( $\mathrm{mm}^{2}$, Fig.1D-G). The VD is the percentage of signal positive pixels per total pixels in an area of interest. FAZ area $\left(\mathrm{mm}^{2}\right)$ was evaluated in the superficial and deep vessel plexus by using the non-flow area tool of the software that delineated it automatically after selecting a segment of the FAZ.

Mapping of the GCC were performed by the $3 \star 3 \mathrm{~mm}$ scan. Eyes were divided in two sectors, superior and inferior. GCC was expressed as the average thickness (in $\mu \mathrm{m}$ ) of both sectors (AvgGCC) and the superior (SupGCC) and inferior (Inf GCC) sectors (Fig.1C).

\section{Statistical Analysis}

Statistical analyses were performed using version 18.0 SPSS statistical software (SPSS, Inc., Chicago, IL, USA). Comparative analyses of three or more groups were made using a one-way analysis of variance (ANOVA), followed by Bonferroni post-hoc tests. Two groups were compared using one- and two-tailed ttests with Bonferroni's corrections. Statistical significance was set at $p<0.05$.

\section{Results}

The mean FAZ are in the SRL was $0.29 \pm 0.05 \mathrm{~mm}^{2}$ in the control group, $0.37 \pm 0.11 \mathrm{~mm}^{2}$ in the NDR group, and $0.41 \pm 0.12 \mathrm{~mm}^{2}$ in the DR group (Fig.2 and Table2). In both the NDR and DR groups, the 
mean FAZ areas were significantly enlarged compared with that of the control group $(F=5.203, P=0.008)$. The mean FAZ area in the DL was $0.55 \pm 0.14 \mathrm{~mm}^{2}$ in the control group, $0.78 \pm 0.29 \mathrm{~mm}^{2}$ in the NDR group, and $0.79 \pm 0.27 \mathrm{~mm}^{2}$ in the DR group (Fig.2 and Table2). The FAZ area in the DL was also significantly enlarged in both the NDR and DR groups compared with the control group ( $F=4.712$, $P=0.012$, Fig. 2 and Table2). No significant difference was observed between the NDR and DR groups for the SRL and DL FAZ area ( $p=0.870$ and 0.570 , Fig.2 and Table2).

In the SRL, the mean (SD) VD\% ratio was $13.43 \% \pm 5.24 \%$ in the control group, $12.56 \% \pm 4 \%$ in the NDR group, and $11.84 \% \pm 2.77 \%$ in the DR group. In both the NDR and DR groups, the mean VD\% was significantly decreased compared with that of the control group $(F=4.683, P=0.034)$. No significant differences were observed between the NDR and DR groups ( $F=1.879, P=0.176$, Fig. 3 and Table2). In the $\mathrm{DL}$, the mean (SD) VD\% ratio was $28.93 \% \pm 4.65 \%$ in the control group, $24.65 \% \pm 3.82 \%$ in the NDR group, and $23.61 \% \pm 4.65 \%$ in the DR group. No significant difference was observed between the NDR and DR groups ( $F=3.411, p=0.070$, Fig.3 and Table2). However, the mean VD\% was statistically significantly lower in patients with $\mathrm{DR}$ in the $\mathrm{DRL}(\mathrm{F}=6.095, \mathrm{P}=0.004$, Fig.3 and Table2 $)$.

Table 2. The FAZ, VD\%, GCC thickness between different group

The mean SupGCC and InfGCC were $112.53 \pm 6.62 \mu \mathrm{m}, 111.6 \pm 6.87 \mu \mathrm{m}$ in the control group, $104.27 \pm 9.59$ $\mu \mathrm{m}$ and $101.70 \pm 14.53 \mu \mathrm{m}$ in the NDR group, and $104 \pm 13.54 \mu \mathrm{m}$ and $97.65 \pm 9.27 \mu \mathrm{m}$ in the DR group. The SupGCC and InfGCC were significantly decreased in both the NDR and DR groups compared with the control group $(F=3.478, P=0.036$ and $F=7.219, P=0.001)$. No significant difference in the SupGCC and the InfGCC was observed between the NDR and DR groups ( $p=0.922$ and 0.170$)$.

Multivariable linear regression was used to study the association of FAZ area and vascular density with AvgGCC in all diabetic eyes. The result showed 2 significant prognostic factors for AvgGCC: FAZ area $(b=-11.372, p=0.001)$ and VD\% $(b=0.752, p=0.007)$ in the $D L$.

We also investigated the interaction between the variables that were found to be significantly associated with worse VA. We found that there were significant interactions with the AvgGCC, FAZ area VD\% $(b=0.752, p=0.007)$ in the $D L$ in the $D R$ group $(R=-0.131, P<0.001, R=-0.049, P=0.037, R=-0.068, P=0.042$, respectively).

\section{Discussion}

In patients with NDR and DR, the mean FAZ areas were increased in comparison to the control group in the SRL and DL. Previous studies have found that significant quantitative differences in the dimensions of the FAZ in DR compared to normal controls $[12,13]$. In these studies, a larger FAZ in DR eyes was demonstrated when analysis the vascular irregularity degree. Mainly, the variations of vascular abnormalities were more noticeable in the deep capillary plexus. An increase of FAZ in DM patients without DR has also been reported before $[5,14]$. These findings suggest compromised circulation in the inner retinal layers before DR manifests. Meanwhile, it's demonstrated the potential value of OCTA as a 
novel angiography technique to detect the early microvasculature structure changes in patients with diabetes.

Compared to normal controls, diabetic patients commonly have a significantly lower capillary perfusion density. Some previous reports have indicated that microaneurysms were present to a larger extent in the deep vascular plexus than in the superficial plexus[15,16]. It has also been suggested that ischemia at the deep capillary layer may play an important role in the changes of the outer retina detected with spectral-domain OCT[15] . In our study, no great changes were detected between SRL and DL. This may be due to the more projection artifacts in detecting the DL structure. Presently, a large number of approaches are being studied to eliminate the overhead shadows impact on deep capillary structures.

The SupGCC and InfGCC were significantly decreased in both the NDR and DR groups compared with the control group. Additionally, in a previous study that included 45 diabetic eyes without retinopathy and 21 non-diabetic eyes significant GCC thinning in diabetes preceded retinal vasculopathy[17] .

Previous studies showed that the originally clinical presentations of diabetic retinal vasculopathy may be the proceeding of functional (decreased contrast sensitivity and color vision) and structural impairment and retinal nerve fiber layer (RNFL) impair before the nonglaucomatous optic neuropathy[18] . van Dijk et al had carried a study to determine the most affected retinal layers in diabetes[19]. It was found that DM causes decreased RNFL thickness in the peripheral macular secondary to the selective ganglion cell layer thinning in the pericentral area. These results backing the conception that diabetes has an early neurodegenerative effect on the retina. Lima et al also found that the GCC was thinner in type 2 diabetic patients in comparison with the non-diabetic controls[20] . Our study showed that SupGCC and InfGCC were significantly decreased in DM groups and AvgGCC thickness was related with FAZ area and VD\% in the DL.

It is now reported that DR is the result of a structural and functional change in the retinal neurovascular unit (NVU)suggesting that neuronal dysfunction and neurodegeneration are closely correlated with microvascular dysfunction and should be considered important components of the pathology of DR[21, 22] . Studies about the retinas of diabetic animal models have demonstrated that retinal neuronal cell degeneration or apoptosis proceeds early in the course of diabetes[23, 24] . A research on intra-retinal layer thickness alterations using optical coherence tomography (OCT) has also indicated that neuroretinal alterations might occur prior to retinal microvascular changes[23] . With the development of OCT and OCTA, it is possible to further reveal their relationship.

There were some limitations of the current study. First, the small sample size for the diabetes with DR group could have limited the potential associations. Selection bias could also have occurerd as a result of choosing a small group. We only included subjects with good VA and OCT images with good quality. Further work is needed to estimate the intra-retinal layer thicknesses in eyes with DME or severe DR with poor fixation. 


\section{List Of Abbreviations:}

DR: Diabetic retinopathy; FA: Fluorescein angiography; OCTA: optical coherence tomography angiography; FAZ: foveal avascular zone; GCC: ganglion cell complexखVD: vessel density; BCVA: bestcorrected VA; NPDR: nonproliferative diabetic retinopathy; RNFL: retinal nerve fiber layer; NVU: neurovascular unit.

\section{Declarations}

Ethics approval and consent to participate: The Declaration of Helsinki was strictly followed in all procedures. Written informed consent, which was approved by the Office of Research Ethical Committee of the Shanghai Jiao Tong University Affiliated Sixth People's Hospital, was obtained from all subjects. It was registered in the Chinese clinical trial registry (http://www.chictr.org.cn/,Registration number: ChiCTROOC-16010160).

Consent for publication: Not applicable.

Availability of data and materials:The datasets used and/or analysed during the current study available from the corresponding author on reasonable request.

Competing interests: The authors declare that they have no competing interests.

Funding: Supported by the Science and Technology Commission of Shanghai Municipality, China (Grant No. 16DZ0501100) and Supported by College-level Project Fund of Shanghai Jiaotong University Affiliated Sixth People's Hospital (Grant No. ynlc201723)

Authors' contributions: Conceived and designed the experiments: XW, QW. Performed the experiments: XW, SL,WQ. Analysed the data: XD. Contributed reagents/materials/analysis tools: SL. Wrote the paper: $X W$. Discussion of the results and critical review of the manuscript: SL. All authors have read and approved the manuscript, and ensure that this is the case.

Acknowledgements: Not applicable.

Authors' information: ${ }^{1}$ Department of Ophthalmology, Shanghai Jiaotong University Affiliated Sixth People's Hospital, 600 Yishan Road, Shanghai 200233, China. ${ }^{2}$ Shanghai Key Laboratory of Diabetes Mellitus, Shanghai 200233, China.

\section{References}

1. Klein R, Klein BE, Moss SE, Davis MD, DeMets DL. The Wisconsin Epidemiologic Study of Diabetic Retinopathy. VI. Retinal photocoagulation. Ophthalmology. 1987; 94:747-53. 
2. Miwa Y, Murakami T, Suzuma K, Uji A, Yoshitake S, Fujimoto M, Yoshitake T, Tamura Y, Yoshimura N. Relationship between Functional and Structural Changes in Diabetic Vessels in Optical Coherence Tomography Angiography. Sci Rep. 2016;6:29064; doi: 10.1038/srep29064.

3. Lee J, Rosen R. Optical Coherence Tomography Angiography in Diabetes. Curr Diab Rep. 2016;16:123.

4. Samara WA, Say EA, Khoo CT, Higgins TP, Magrath G, Ferenczy S, Shields CL. Correlation of foveal avascular zone size with foveal morphology in normal eyes using optical coherence tomography angiography. Retina. 2015;35:2188-95.

5. Di G, Weihong Y, Xiao Z, Zhikun Y, Xuan Z, Yi Q, Fangtian D. A morphological study of the foveal avascular zone in patients with diabetes mellitus using optical coherence tomography angiography. Graefes Arch Clin Exp Ophthalmol. 2016;254:873-9.

6. Freiberg FJ, Pfau M, Wons J, Wirth MA, Becker MD, Michels S. Optical coherence tomography angiography of the foveal avascular zone in diabetic retinopathy. Graefes Arch Clin Exp Ophthalmol. 2016;254:1051-8.

7. Bowd C, Tafreshi A, Zangwill LM, Medeiros FA, Sample PA, Weinreb RN. Pattern electroretinogram association with spectral domain-OCT structural measurements in glaucoma. Eye (Lond). 2011;25:22432.

8. Gardner TW, Abcouwer SF, Barber AJ, Jackson GR. An integrated approach to diabetic retinopathy research. Arch Ophthalmol. 2011;129:230-5.

9. Barber A J. Diabetic retinopathy: recent advances towards understanding neurodegeneration and vision loss. Sci China Life Sci. 2015;58(6):541-9.

10. Jonsson K B, Frydkjaer-Olsen U, Grauslund J. Vascular Changes and Neurodegeneration in the Early Stages of Diabetic Retinopathy: Which Comes First?. Ophthalmic Res.2016;56:1-9.

11. Wilkinson C P, Ferris F R, Klein R E, et al. Proposed international clinical diabetic retinopathy and diabetic macular edema disease severity scales. Ophthalmology. 2003;110:1677-82.

12. Villarroel M, Ciudin A, Hernandez C, Simo R. Optical coherence tomography angiography of the foveal avascular zone in diabetic retinopathy. Graefes Arch Clin Exp Ophthalmol. 2016;254:1051-8.

13. Takase N, Nozaki M, Kato A, Ozeki H, Yoshida M, Ogura Y. Engargement of foveal avascular zone in diabetic eyes evaluated by en face optical coherence tomography angiography. Retina. 2015;35:2377-83.

14. de Carlo T E, Chin A T, Bonini F M, et al. Detection of microvascular changes in eyes of patients with diabetes but not clinial diabetic retinopathy using optical coherence tomography angiography. Retina. 2015;35:2364-70. 
15. Scarinci F, Jampol LM, Linsenmeier RA, Fawzi AA. Association of Diabetic Macular Nonperfusion With Outer Retinal Disruption on Optical Coherence Tomography. JAMA Ophthalmol. 2015;133:1036-44.

16. Couturier A, Mane V, Bonnin S, Erginay A, Massin P, Gaudric A, Tadayoni R. Capillary plexus anomalies in diabetic retinopathy on optical coherence tomography angiography. Retina. 2015;35:2384-91.

17. Hegazy Al, Zedan RH, Macky TA, Esmat SM. Retinal ganglion cell complex changes using spectral domain optical coherence tomography in diabetic patients without retinopathy. Int $\mathrm{J}$ Ophthalmol. 2017;10:427-33.

18. Kurtenbach A, Flogel W, Erb C. Anomaloscope matches in patients with diabetes mellitus. Graefes Arch Clin Exp Ophthalmol. 2002; 240:79-84

19. van Dijk H W, Verbraak F D, Kok P H, Garvin MK, Sonka M, Lee K, Devries JH, et al. Decreased retinal ganglion cell layer thickness in patients with type 1 diabetes. Invest Ophthalmol Vis Sci. 2010;51:3660-5.

20. Lima V C, Prata T S, Pacheco M A, et al. Macular Inner Retinal Layer Thinning in Diabetic Patients Without Retinopathy Measured by Fourier Domain Optical Coherence Tomography. Investigative Ophthalmology \& Visual Science. 2010;51:341.

21. Antonetti D A, Klein R, Gardner T W. Diabetic retinopathy. N Engl J Med. 2012;366:1227-39.

22. Abcouwer S F, Gardner T W. Diabetic retinopathy: loss of neuroretinal adaptation to the diabetic metabolic environment. Ann N Y Acad Sci. 2014;1311:174-90.

23. Chhablani J, Sharma A, Goud A, Peguda HK, Rao HL, Begum VU, et al. Neurodegeneration in Type 2 Diabetes: Evidence From Spectral-Domain Optical Coherence Tomography. Invest Ophthalmol Vis Sci. 2015;56:6333-8.

24. van Dijk H W, Verbraak F D, Kok P H, Stehouwer M, Garvin MK, Sonka M, et al. Early neurodegeneration in the retina of type 2 diabetic patients. Invest Ophthalmol Vis Sci. 2012;53:2715-9.

\section{Tables}

Table 1. Characteristics of Patients

\begin{tabular}{|c|c|c:c|}
\hline Characteristics & Control group & NDR group & DR group \\
\hline No.patients & 22 & 30 & 34 \\
\hline No.eyes & 22 & 30 & 34 \\
\hline Age,Y & $62.87 \pm 9.82$ & $63.16 \pm 8.49$ & $62.59 \pm 9.62$ \\
\hline Gender (male/female) & $10 / 12$ & $14 / 16$ & $19 / 15$ \\
\hline Laterality (right/left) & $12 / 10$ & $13 / 17$ & $20 / 14$ \\
\hline BCVA, logMAR* & $0.12 \pm 0.12$ & $0.15 \pm 0.15$ & $0.35 \pm 0.27$ \\
\hline HbA1c,\%** & & $7.2 \pm 1.5$ & $9.1 \pm 2.3$ \\
\hline Axial legth, mm & $23.16 \pm 1.72$ & $23.80 \pm 1.56$ & $23.72 \pm 1.48$ \\
\hline Refractive error & $0.26 \pm 1.82$ & $0.59 \pm 2.12$ & $0.41 \pm 1.63$ \\
\hline
\end{tabular}


BMI: body mass index; BCVA: best corrected visual acuity.

* Significant difference among group means via one-way ANOVA $(\mathrm{P}<0.001)$

** Significant difference the two group means via t-test $(\mathrm{P}<0.001)$

Table 2. The FAZ, VD\%, GCC thickness between different group

\begin{tabular}{cccccc}
\hline & & Control group & NDR group & DR group & P* \\
\hline FAZ & SRL & $0.29 \pm 0.05 \mathrm{~mm}^{2}$ & $0.37 \pm 0.11 \mathrm{~mm}^{2}$ & $0.41 \pm 0.12 \mathrm{~mm}^{2}$ & 0.008 \\
& DL & $0.55 \pm 0.14$ & $0.78 \pm 0.29 \mathrm{~mm}^{2}$ & $0.79 \pm 0.27 \mathrm{~mm}^{2}$ & 0.012 \\
& & $\mathrm{~mm}^{2}$ & & & \\
VD\% & SRL & $13.43 \% \pm 5.24 \%$ & $12.56 \% \pm 4 \%$ & $11.84 \% \pm 2.77 \%$ & 0.034 \\
& DL & $28.93 \% \pm 4.65 \%$ & $24.65 \% \pm 3.82 \%$ & $23.61 \% \pm 4.65 \%$ & 0.070 \\
GCC & SupGCC & $112.53 \pm 6.62 \mu \mathrm{m}$ & $104.27 \pm 9.59 \mu \mathrm{m}$ & $104 \pm 13.54 \mu \mathrm{\mu m}$ & 0.036 \\
thickness & InfGCC & $11.6 \pm 6.87 \mu \mathrm{m}$ & $101.70 \pm 14.53$ & $97.65 \pm 9.27 \mu \mathrm{m}$ & 0.001 \\
& \multicolumn{5}{c}{$\mu \mathrm{m}$} \\
\hline
\end{tabular}

* Significant difference between the control group and (NDR\&DR)group via t-test $(\mathrm{P}<0.05)$

\section{Figures}


Thickness Map( ILM - RPE/BM )

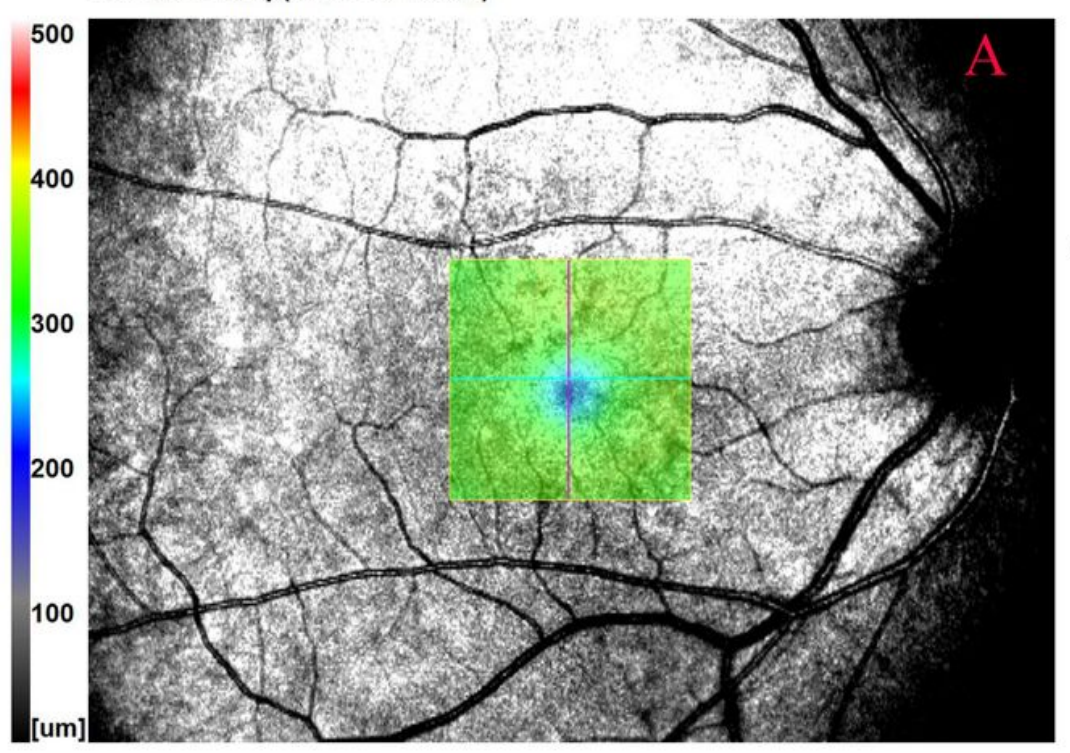

JCT( Horizontal 128 / 256 )

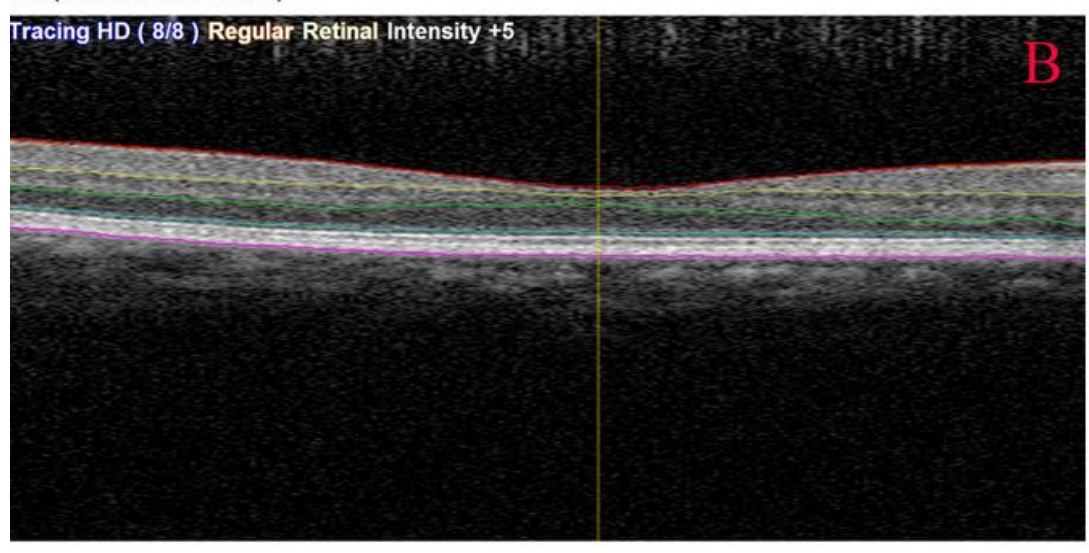

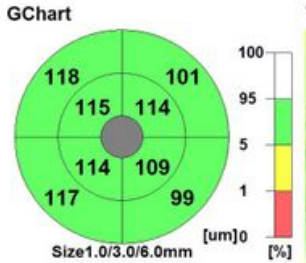

ThicknessMap( ILM - IPLINL)

$\mathrm{S} /$
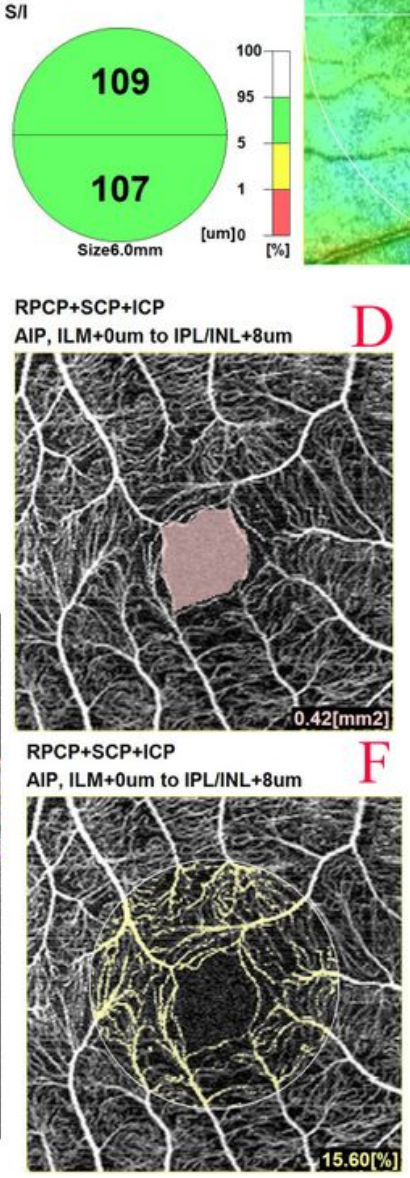

DCP

AIP, IPL/NL+12um to IPL/INL+87um
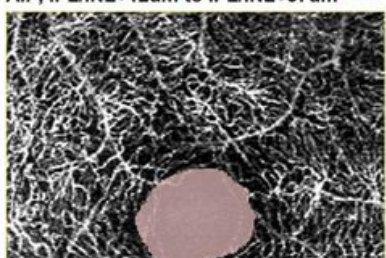

sig
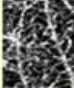

DCP

AIP, IPL/NL+12um to IPL/INL+87um

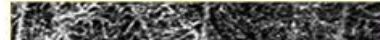

8

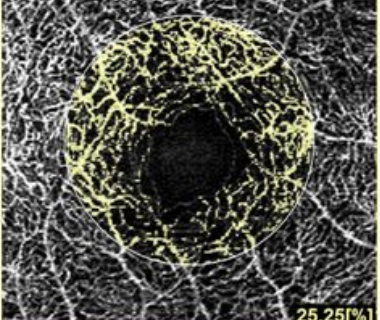

Figure 1

FAZ and macular VD measurement using OCTA. The $3 \times 3-\mathrm{mm}$ OCTA scans are centered on the macula(A and $B$ ). An automated software algorithm integrated into the OCTA system graphically highlighted and calculated the FAZ area in the superficial (D) and deep (E) networks. Vessel density (VD\%) is also calculated in the superficial $(F)$ and deep (G) networks. Segmentation of the foveal ganglion cell layer thickness was calculated (C). 


\section{Foveal avascular zone}

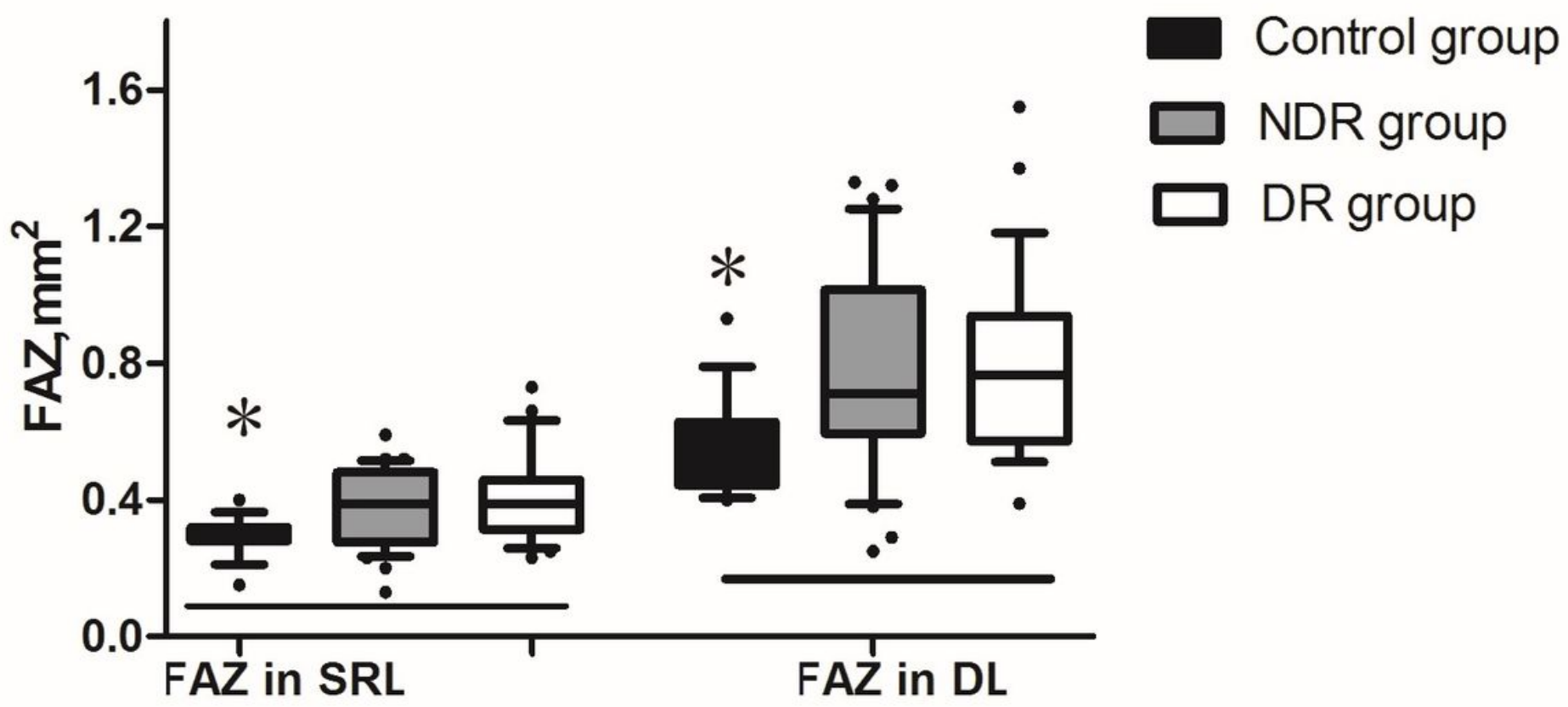

Figure 2

Boxplot showing the FAZ area in the SRLs and DRLs in control groups, patients with DR and subgroups of patients with NDR. 


\section{Vascular density}

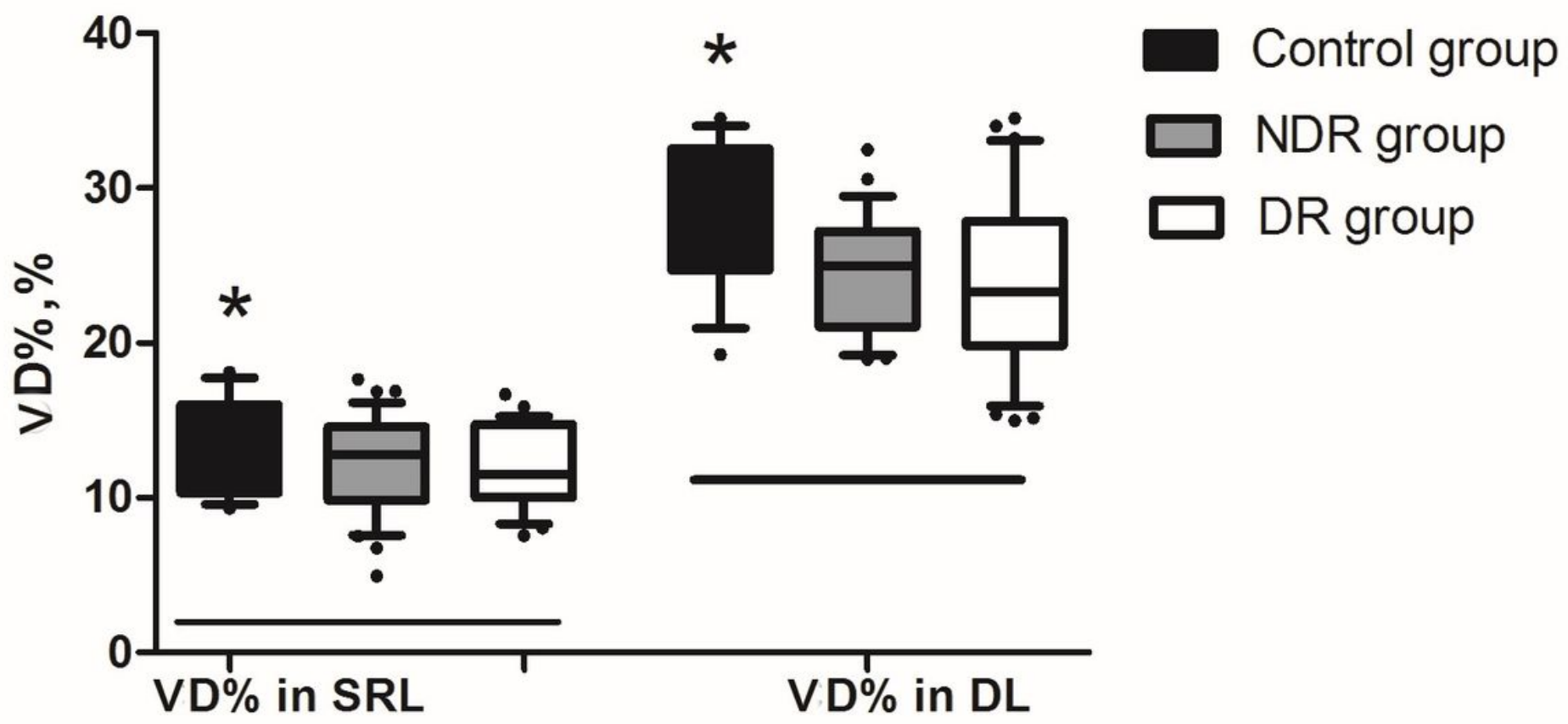

Figure 3

Boxplot showing the vascular density in the SRLs and DRLs in control group, patients with DR and subgroups of patients with NDR. 